\title{
ASENTAMIENTO Y DINÁMICA POBLACIONAL OLMECA EN LA REGIÓN DE CAPOACAN, VERACRUZ
}

\section{SETTLEMENT AND OLMECA POPULATION DYNAMICS IN THE REGION OF CAPOACAN, VERACRUZ}

\section{Virginia Arieta Baizabal ${ }^{1}$}

varieta@uv.mx

\section{RESUMEN}

Antonio Plaza, ubicado al margen del río Uxpanapa, es conocido y señalado como el lugar de origen de uno de los hallazgos más polémicos de la arqueología de la costa del Golfo, hacemos referencia a la escultura conocida como El Luchador. A pesar de que esta extraordinaria pieza ha provocado la discusión entre académicos, durante décadas, sobre su autenticidad arqueológica, no se había realizado una investigación sistemática arqueológica en la región de Capoacan -un área estratégica intermedia entre los ríos Coatzacoalcos, Capoacan y Uxpanapa; una isla ubicada justo en medio de los sitios olmecas de San Lorenzo, Veracruz y La Venta, Tabasco. Por tal motivo, en 2018 dio inicio un proyecto sistemático en la región que tiene como uno de sus objetivos la clarificación de hechos y la contribución al conocimiento de la arqueología regional. En este artículo se presentarán los resultados de la primera temporada de campo y análisis de los materiales, mismos que revelan información importante sobre la dinámica poblacional de un sitio intermedio, espacial y temporalmente, a las dos capitales olmecas del periodo Preclásico mesoamericano.

Palabras clave: Olmecas, patrón de asentamiento, dinámica poblacional, interacción.

\footnotetext{
${ }^{1}$ Instituto de Antropología, Universidad Veracruzana, México.
} 


\begin{abstract}
Antonio Plaza, on the Uxpanapa river bank, is known as the place of origin of one of the most controversial archaeological findings in the Southern Gulf of Mexico - the so-called Wrestler sculpture. Despite the discussion on its archaeological authenticity that, for decades, this extraordinary piece has sparked, no thorough archaeological research has been undertaken in the region of Capoacan - a strategic area between the rivers Coatzacoalcos, Coachapa and Uxpanapa; an island located just between the Olmec sites of San Lorenzo, Veracruz and La Venta, Tabasco. For this reason, 2018 saw the launch of a systemaci research project in the aforementioned region, whose principal aim is to clarify facts and contribute to the body of knowledge on the region's archaeological heritage. This paper presents the findings after the first season of field work and the analysis of the recovered archaeological material, which reveal important information on the population dynamics in an intermediate - both in space and time - site between the two major Olmec capitals of the Mesoamerican Pre-classic period.
\end{abstract}

Keywords: Olmec, settlement pattern, population dynamics, interaction

\title{
CONTEXTO DE LA INVESTIGACIÓN
}

El sur de Veracruz es quizá el área, dentro de la costa del Golfo de México, más investigada a través de estudios enfocados en el patrón de asentamiento (Drucker y Contreras 1953; Kruger 1996; Borstein 2001; Symonds, Cyphers y Lunagómez 2002; Alonso 2003; Wendt 2005; Jiménez 2008, Lunagómez 2014, Arieta 2018). A los antes mencionados, hay que agregar los resultados de la supervisión arqueológica en obras de trabajo sísmico (Hernández 2007, 2008, 2010 y 2012). Dichas investigaciones han modificado la perspectiva, demostrando la viabilidad de establecimiento en el trópico desde época prehispánica. 
Específicamente, los trabajos realizados en la capital de San Lorenzo han expuesto la preferencia de la sociedad olmeca por el asentamiento en terrenos elevados, así como en sitios particulares dentro de humedales, tal es el caso de los islotes, utilizados para obtener alimentos acuáticos y complementar su dieta basada en tubérculos (Symonds, Cyphers y Lunagómez 2002; Cyphers, Zurita y Lane 2013). También, se ha obtenido información sobre las estrategías de almacenamiento, el manejo del riesgo en áreas de inundación anual, intercambio, comunicación, transporte y movilidad de los antiguos pobladores de las llanuras costeras a través de un puerto fluvial conectado por imponentes ríos caudalosos de México (Cyphers y Hirth 2016). Finalmente, y a pesar de no ser facilmente identificables y detectables los restos arqueológicos habitacionales del periodo Preclásico -debido a su considerable profundidad de hasta 20 metros-, los estudios sobre tendencias demográficas en San Lorenzo han demostrado la importancia de comprender las diversas y complejas dinámicas poblaciones que se desarrollaron en el trópico (Arieta 2013; Arieta y Cyphers 2017; Arieta y Cyphers en prensa). En resumen, actualmente sabemos que el peculiar medio ambiente de la selva húmeda tropical, que caracteriza al sur de la costa del Golfo de México, definido por entornos acuosos de pantano y popal, contribuyó y fue clave para el desarrollo de sociedades como lo es la olmeca en el periodo Preclásico.

A pesar de lo antes descrito, no se había realizado una investigación sistemática arqueológica en un área particular dentro del sur de Veracruz, nos referimos a la región de Capoacan,-un área intermedia entre el río Coatzacoalcos, el río Coachapa y la cuenca alta del río Uxpanapa; una isla ubicada a tan sólo a $35 \mathrm{~km}$ al 
noreste de la capital olmeca de San Lorenzo y a $44 \mathrm{~km}$ al suroeste de la capital olmeca de La Venta, Tabasco (Figura 1). Fue hasta el año 2017 que dio inicio un proyecto de investigación arqueológica sistemática que comenzó con una temporada de recorrido de superficie en la región antes mencionada, teniendo como objetivo conocer el patrón de asentamiento y la dinámica poblacional de los antiguos habitantes, la clarificación de hechos sobre esculturas halladas en la décadas previas y la contribución al conocimiento de la arqueología regional. De tal forma que la información aquí expuesta complementa y extiende la cobertura al añadise a los estudios en el sur de Veracruz arriba mencionados. El presente trabajo tratará de describir el patrón de asentamiento del periodo Preclásico en el área de Capoacan, así como explicar la dinámica poblacional y el vínculo sociopolítico entre los sitios olmecas de la región y las dos capitales de San Lorenzo, Veracruz y La Venta, Tabasco.



Figura 1. Ubicación geográfica de la Isla de Capoacan-sitio Antonio Plaza y su distancia respecto a las capitales olmecas de San Lorenzo y La Venta. Elaborado por Virginia Arieta. 


\section{UN SITIO ARQUEOLÓGICO INEXPLORADO: ANTONIO PLAZA, VERACRUZ}

Antonio Plaza, antes llamado San María de Uxpanapa, es una localidad ubicada dentro de la región de Capoacan, a escasos $12 \mathrm{~km}$ en línea recta del municipio de Minatitlán, al sur de la costa del Golfo de México. Específicamente, se encuentra en una elevación intermedia de la llamada "Isla de Capoacan" -un terreno con una elevación máxima de $42 \mathrm{msnm}$ que favorece el asentamiento a salvo de las inundaciones anuales, resultado del desbordamiento de los ríos que rodean el área: río Coatzacoalcos, río Coachapa y río Uxpanapa.

La localidad de Antonio Plaza es conocida debido a que se trata del supuesto lugar de hallazgo de una de las esculturas más polémicas de la arqueología de la costa del Golfo. Hacemos referencia a la famosa pieza de basalto conocida como El Luchador de Antonio Plaza o El Luchador del Uxpanapa, llamada así por representar a un personaje con los brazos levantados y girando el torso, causando la impresión de movimiento. Durante las últimas décadas, opiniones con relación a su autenticidad de pieza arqueológica y por ende, a su pertenencia a la cultura olmeca, han estado divididas entre quienes sostienen que se trata de una falsificación moderna (Kelker 2004; Kelker y Bruhns 2010) y quienes piensan que es auténtica y típicamente olmeca (Stirling 1945; Coe y Miller 2005; Cyphers y López 2007, 2008). Hasta el momento, la postura mejor fundamentada es la propuesta por Cyphers y López (2007:70) quienes, a través de un estudio etnográfico en torno a los diversos dueños de la escultura, consiguieron relatar la 
historia de la pieza, desde su aparición hasta su permanencia actual como parte de la colección del Museo Nacional de Antropología desde 1964, validando de forma documentada su autenticidad. Es de suma importancia mencionar para lo objetivos de la presente investigación que en la publicación de Cyphers y López (2008), sobre la historia reciente de El Luchador, se menciona, con base al material arqueológico observado en la localidad durante el estudio etnográfico, la ocupación principal del sitio arqueológico de Antonio Plaza es durante el periodo Preclásico medio (1000-700 aC). Los autores concluyen con la necesidad y urgencia de un estudio sistemático en la isla de Capoacan y el área circundante con la finalidad de conocer el papel del sitio arqueológico de Antonio Plaza en época olmeca.

Como arriba hemos mencionado, es hasta el año 2017 que da inicio el Proyecto Arqueológico Antonio Plaza-Capoacan (PAAPC) teniendo como principal objetivo identificar la presencia o ausencia de ocupación prehispánica, es decir, inferir en primer lugar si en efecto se trata de un sitio arqueológico; consecuentemente, conocer el patrón de asentamiento y reconstruir la dinámica poblacional de la región de Capoacan, tomando como centro a la localidad de Antonio Plaza, a través de un programa de reconocimiento de superficie. En este trabajo exponemos los primeros resultados, derivando información inédita e importante de un sitio inexplorado en el contexto de una arqueología regional. 


\section{PERSPECTIVAS TEÓRICAS}

Estamos plenamente convencidos que en la investigación arqueológica, a la par de la implementación de novedosas tecnologías, debe de estar la base teórica. Por tanto y dado que el estudio del patrón de asentamiento es uno de nuestros objetivos principales, la perspectiva en la que se enmarca el presente estudio es el de la Ecología cultural. Consecuentemente y de manera conjunta, se plantea el análisis de la dinámica poblacional y su organización socio-política, por lo tanto, también nos servimos de los presupuestos del enfoque de la Arqueología demográfica. A continuación se describirán brevemente los planteamientos de ambos enfoques y cómo su interrelación resulta enriquecedora para los fines de la presente investigación.

Gonzáles Crespo (1979) menciona que, si bien es ciertos, los estudios de patrón de asentamiento fueron propuestos por Sanders, la primera aplicación fue efectuada por Willey en 1953 en el valle de Virú, Perú. Estudios de esta naturaleza fueron rápidamente aceptados, acentuándose con el desarrollo de la Arqueología Procesual o la Nueva Arqueología de los años sesenta y setenta, que plantea y define a los grupos humanos como sistemas culturales completos y abiertos, sujetos a los estímulos de diversos factores adherentes a la sociedad, principalmente el factor medio ambiental -aunque hay en reconocer que la propuesta en torno a la importancia de la relación entre el medio ambiente y la cultura fue hecha por los geógrafos culturales desde la década los treinta (Sauer 1935)-. De acuerdo a lo anterior, los arqueólogos se percataron de la importancia 
en la corriente de la Ecología cultural para llegar a la interpretación de la dinámica de las sociedades y la relación con su entorno.

En el caso de Mesoamérica, la perspectiva fue aplicada por el mismo Willey (et al 1965) en el valle de Belice a finales de la década de los cincuenta y por Sanders, Parsons y Santley (1979) en el valle de México. Desde entonces, los arqueólogos han reafirmado la posición del estudio del patrón de asentamiento como el complemento para la comprensión de las relaciones sociales de comunidades antiguas. Hoy en día, este tipo de estudios se han vuelto imprescindibles a la hora de formular estrategias de investigación y en el proceso de interpretación de modos de vida en el pasado.

En el sur de la costa del Golfo de México, los estudios de patrón de asentamiento tienen una larga historia (Drucker y Contreras 1953, Gómez Rueda 1996, Stark 2001, Santley et al 1992, Kruger 1996, Borstein 2001, Daneels 2002, Pool 2000, 2003, Killion y Urcid 2001, Esquivas 2002, Loughlin 2004 y Budar 2014). Especificamente, cercanos a la región de Capoacan se encuentran los de Lunagómez 2014, Jiménez 2008, Wendt y Lunagómez 2011. Asismismo, de especial interés para esta propuesta de investigación por cercanía al área, se encuentra la investigación regional sistemática más amplia del sur de Veracruz. Nos referimos a la efectuada en el área de San Lorenzo, durante 1992 y 1994, por parte del "Proyecto Arqueológico San Lorenzo, Tenochtitlán" (PASLT) a cargo de Ann Cyphers. El reconocimiento cubrió un área de $700 \mathrm{~km}^{2}$, reportando más de 250 sitios y proponiendo cálculos de densidad poblacional y alternativas de 
subsistencia (Lunagómez 1995, 2011 y Symonds, Cyphers y Lunagómez 2002). A este último, se añade el estudio de Alonso (2003) quien realiza un reconocimiento de superficie al sur del hinterland de San Lorenzo.

Por su parte, la perspectiva de la Arqueología demográfica proporciona evidencias básicas sobre el patrón de asentamiento a nivel intra-sitio (relación de unidades domésticas con otro tipo de estructura ceremonial o religiosa) y unidades domésticas. Este enfoque tiene como propósito fundamental realizar modelos de explicación acerca de los diversos cambios que ha sufrido la sociedad humana, en particular aquellos relacionados con las características demográficas y poblaciones con relación al entorno. Por lo tanto, este modelo teoricometodológico surge de la necesidad de crear hipótesis que expliquen cómo estaba conformada la estructura poblacional de sociedades antiguas y cómo ésta se refleja a través de los patrones demográficos estáticos, como lo son la distribución espacial, tendencias de crecimiento, cohesión, expansión, tamaño, densidad, entre otros. El resultado no sólo será el cálculo de tamaño de población o el grado de cohesión o dispersión, sino la interpretación de fenómenos dinámicos de la cultura como la organización social y política, migraciones, subsistencia, relación con el paisaje y entorno, entre otros.

Fue Fekri Hassan, quien adaptó y desarrolló un cuerpo de técnicas y métodos para el estudio demográfico de poblaciones antiguas por medio de datos arqueológicos y que él mismo denomina como Arqueología demográfica (Hassan 1978). El modelo hace hincapié nuevamente en los estudios de patrón de asentamiento a 
distintos niveles y en la reconstrucción del tamaño y densidad de sitios y estructuras arquitectónicas como elementos esenciales para poder conocer la dinámica poblacional. Desde este punto de vista, los estudios de patrón de asentamiento en sus distintos niveles (regional, sitio e intra-sitio) reconocen que las poblacionales no son únicamente conglomerados de individuos, sino sistemas ordenados congruentes que contienen una estructura conformada de características biológicas y culturales dentro de un marco histórico cultural. De esta manera, los enfoques teóricos-metodológicos de la Ecología cultural y la Arqueología demográfica se complementan para poder comprender y explicar la dinámica poblacional antigua en el área de investigación a través de los estudios de patrón de asentamiento. Dichas perspectivas, de acuerdo a las características de la región de estudio y los antecedentes de su aplicación satisfactoria, son los que brindan mayores posibilidades en la explicación de dinámicas poblacionales al incluir la evidencia arqueológica con relación a las particularidades medio ambientales, en este caso las llanuras costeras del sur de Veracruz.

Es de suma importancia mencionar que el presente proyecto se encuentra en la primera etapa de investigación (estudio de superficie y morfometría de los sitios) que abarca todo lo que tiene que ver con la identificación, ubicación y estimación del tamaño de cada sitio a través de la distribución de arquitectura y materiales cerámicos y líticos. De acuerdo a los resultados obtenidos se pretende pasar, en un futuro cercano, a la segunda fase con fines de adquirir indicadores materiales arqueológicos como son el número de estructuras arquitectónicas, las áreas de actividad y restos de alimento, proporcionará información sobre el área de 
ocupación, el crecimiento, desarrollo y densidad de la población extinta a través de un programa de excavaciones estratigráficas. El vínculo entre la perspectiva teórica de la Ecología cultural y la Arqueología demográfica brindarán en conjunto la posibilidad de interpretar un panorama sobre la ocupación prehispánica en la región de Capoacan con una visión integral en el contexto general del sur de la costa del Golfo de México.

\section{CONSIDERACIONES METODOLÓGICAS: PROCEDIMIENTOS DE CAMPO Y LABORATORIO}

La metodología de campo implementada por PAAPC en la región de Capoacan fue la utilizada en otros sitios del sur de Veracruz (Lunagómez 1995 y Symonds et al 2002), aunque se realizaron algunas modificaciones técnicas de acuerdo a un estudio previo de mapeo) y análisis del terreno, tal es el caso de una proyección digital de inundaciones por desborde de ríos y el reconocimiento de zonas con vegetación selvática densa con apoyo de tecnología LiDAR (Light detection and ranging-detección de luz y distancia) Landsat5 2011, Multiespectral 7 bandas, fecha de toma 23/03/2011 (Figura 2). Dicha fase fue significativa porque reveló información proveniente de la superficie terrestre con georregerencia, a bajo costo y $\sin$ la destrucción de vestigios, a través de la identificación de pequeñas diferencias de temperatura, forma y textura en la superficie que estén asociadas a rasgos culturales. Dicha etapa de análisis previo permitieron generar mapas de las áreas cubiertas por vegetación densa, e inundadas y determinar qué zonas necesitan mayor documentación, tal es el caso de los márgenes del río Uxpanapa, caracterizados por ser áreas aluviales. No obstante, es de suma importancia 
mencionar que el área de investigación propuesta se caracteriza por la presencia de vegetación cerrada, popal y pantanos, típicos de la selva húmeda tropical, por lo que muchas anomalías culturales que no fueron identificadas en esta primera fase de mapeo, fueron registradas durante la temporada de campo a través del programa de reconocimiento de superficie. Dicho método de superficie consiste en caminatas en línea recta o transectos a intervalos de separación entre los miembros del equipo con distancia de $20 \mathrm{~m}$, permitiendo ubicar sitios representados con concentraciones o dispersiones de material arqueológico, sin omitir ningun tipo de terreno: pantanos, arroyos, acahuales, sembradíos de maiz altos y bajos, zonas inundables, potreros para ganado.

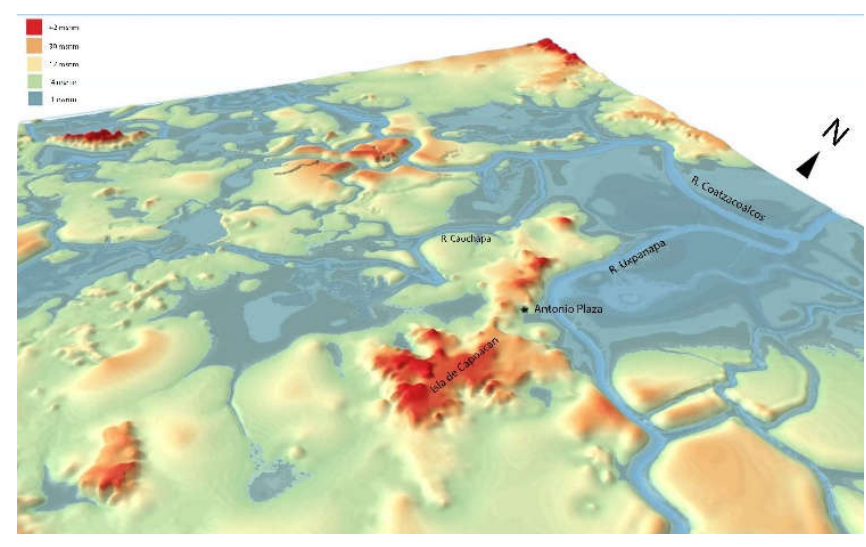

Figura 2. Imagen que muestra una proyección de inundaciones por desborde de ríos en la región de Capoacan. Elaborado por Virginia Arieta con datos INEGI. Landsat5 2011, Multiespectral 7 bandas, fecha de toma 23/03/2011.

Asimismo, es de suma importancia mencionar que de acuerdo con lo observado por otros proyectos de investigación enfocados en sitios del sur de Veracruz, existe una tendencia en la localización de sitios, correspondientes al periodo Preclásico, cerca de los ríos y arroyos. De acuerdo a lo anterior, las zonas en los 
márgenes de cuerpos de agua fueron de principal interés para los objetivos de este proyecto. Por tanto, a pesar de la complejidad que implica llevar a cabo un recorrido sistemático en áreas de inundación y pantano, el recorrido pedestre en transectos a corta distancia permitió la localización y registro de concentraciones de material cerámico como se observará en los resultados. Las colecciones fueron tomadas en la superficie total del sitio y ubicadas en el mapa maestro generado a través de Sistemas de Información Geográfica (SIG), con el fin de conocer la ubicación exacta dentro del sitio. Posteriormente, se tomaban fotografía y fotografía aerea con drone para sitios con arquitectura visible, se ubicaban en la carta topográfica y el mapa maestro a través de SIG. Para la obtención de datos topográficos y cartográficos utilizamos un drone que posibilita la captura de imágenes de muy alta resolución en zonas abiertas y puede apoyar en la producción de cartografía aérea con una precisión de centímetros logrando mostrar la posible existencia de arquitectura prehispánica en superficie.

Posterior al trabajo de campo, el análisis de los materiales arqueológicos trata de una de las etapas metodológica más importante, ya que a través la derivación de datos e información se construyen las inferencias e interpretaciones. La estrategía del PAAPC consistió en una primera separación de tiestos por pasta, desgrasante a nivel microscópico y color Munsell. En los casos posibles la clasificación inicial incluía la presencia de engobe, color de engobe y decoración. Consecuentemente, se denominó a grupos cerámicos derivados y se obtuvieron agrupaciones definidas por el color de la pasta y desgrasante. 
La presente investigación tiene como una de sus metas la aportación de nuevos datos que llenen vacíos sobre el conocimiento de la historia ocupacional prehispánica de esta región en la costa del Golfo. Por tal motivo, pensamos que el establecimiento de tipos cerámicos propios generaría cuantiosa información exclusiva para el área de estudio y no, datos que ayuden al conocimiento de la arqueología regional. De acuerdo con lo anterior, el siguiente paso fue comparar la primera clasificación del PAAPC con tipologías cerámicas propuestas por otros proyectos enfocados áreas cercanas, y de importante relación, sobre todo con sitios con el que pudo haber existido un considerable vínculo durante el periodo Preclásico, como San Lorenzo, Veracruz y La Venta, Tabasco. Cuando la concordancia era clara y segura se le asignaba al grupo, el nombre propuesto.

Dicha correlación representa la base para la asignación de periodos y fases de ocupación a las fases cronológicas ya establecidas, y se logra observar la distribución espacial por tipo en un área mucho más amplia. De esta manera, se obtienen los marcadores cerámicos utilizados para el fechamiento de los sitios: para el Preclásico Inferior se utilizaron las descripciones de los tipos de acuerdo a la clasificación hecha por Cyphers para el Proyecto Arqueológico San LorenzoTenochtitlan (PASLT) (Symonds et al., 2002); para el Preclásico Medio se utilizó la propuesta por Rust (2008) y Von Nagy (et al., 2002); mientras que para el Preclásico Tardío se recurrió en los tipos reportados por Coe y Diehl (1980) para la fase Ramplás; finalmente, para el periodo Clásico se incluyen los tipos registrados por Coe y Diehl (1980) y Symonds et al. (2002). Por supuesto, se identificaron grupos que escapan de las descripciones ya propuestas, a ellos se les 
ha dejado el nombre descriptivo compuesto por las características de la pasta, desgrasante y color Munsell, para distinguirlos y con la finalidad de continuar la documentación bibliográfica respecto a tipologías ya formuladas. Los resultados del trabajo de campo y laboratorio en su conjunto nos llevan a inferir y conocer el patrón de asentamiento de la región de Capoacan a lo largo del tiempo de manera sistemática y confiable.

\section{PATRÓN DE ASENTAMIENTO EN LA REGIÓN DE CAPOACAN}

Tanto el programa de mapeo, como el programa de reconocimiento de superficie, requirieron del uso de SIG para inferir el patrón de asentamiento. Primero, el procesamiento digital de la topografía del área de estudio mediante las aplicaciones $Q G I S$ fue de vital ayuda para la comprensión de la superficie y la ubicación geográfica y gráfica de los sitios. Segundo, el análisis espacial y la elaboración de mapas temáticos sobre delimitación de distancias, alturas, rutas mínimas, accesos, gráficos de distribución de distintas variables, entre otros, fueron de gran apoyo para tener mayor claridad de la ocupación, aprovechamiento del área y el patrón de asentamiento.

Los SIG permiten el análisis de información espacial y temporal para expresar un modelo racional de base de datos de un mismo marco geográfico integrando diferentes variables de análisis. La estimación confiable del tamaño y distribución de los sitios conllevó la generación de Heat maps (mapas de calor) que permiten identificar fácilmente excepciones e información relevante que no es observable a 
simple vista con otro tipo de instrumento y El análisis cuantitativo se llevó a cabo a través de un ejercicio geoestadístico para el cual se utilizó el mismo perímetro para la definición de sitios independientes en la estrategía metodológica de campo (150 metros). A la diferenciación espacial, vista a través del análisis en torno a la variable de distancia, le acompañan dos distinciones más: altitud, secuencia cronológica establecida a través de las tipologías cerámicas de regiones vecinas y características arquitectónicas (Figura 3 y 4).

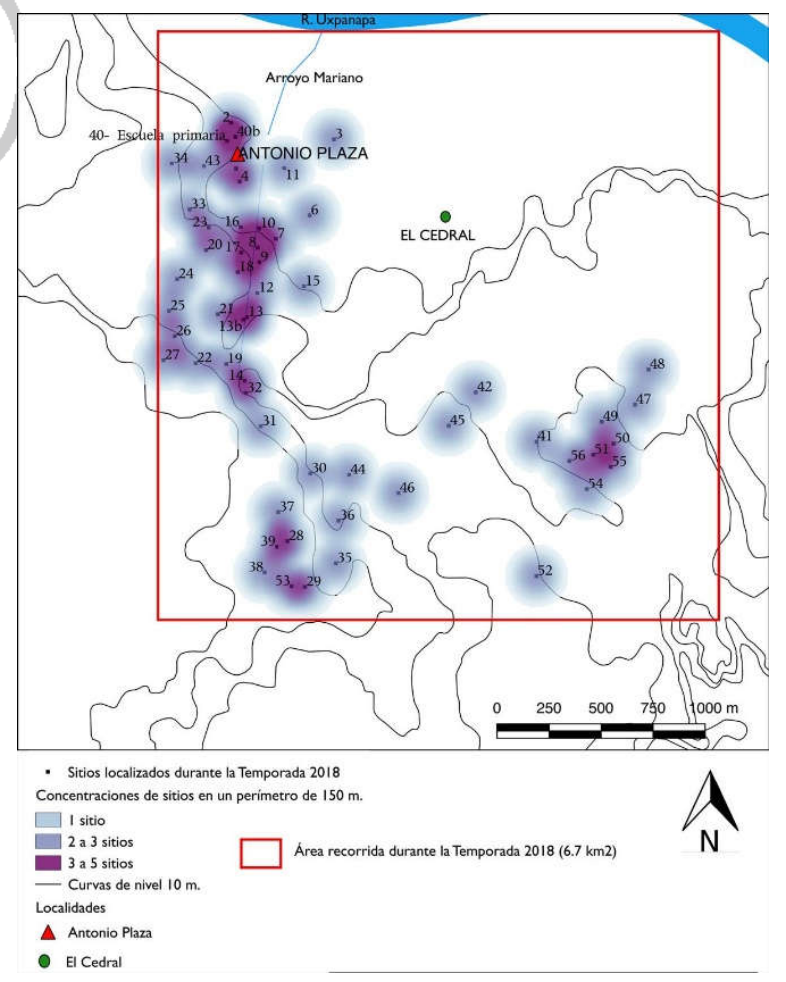

Figura 3. Imagen que muestra las agrupaciones de concentraciones de material arqueológico respecto al valor de las varias geoespaciales: elevación y distancia. Elaborado por Virginia Arieta. 


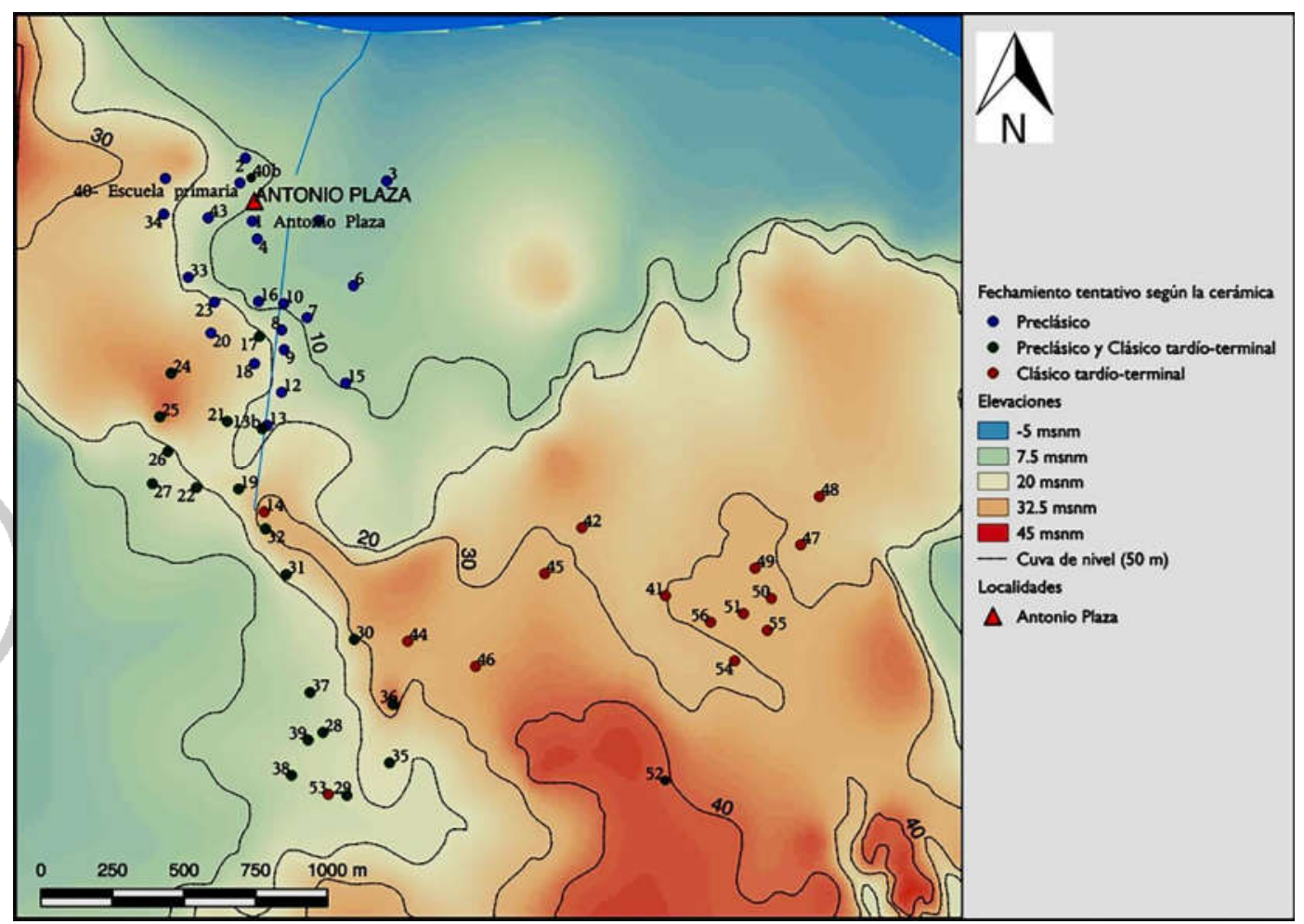

Figura 4. Imagen que muestra las agrupaciones de concentraciones de material arqueológico respecto a los resultados del análisis cerámico. Elaborado por Virginia Arieta.

Durante la temporada 2018, se identificaron 56 concentraciones de material arqueológico (cerámica, lítica tallada y lítica pulida). En este caso, el análisis de la distribución basandonos en las variables arriba mencionadas (distancia, altitud, cronología establecida como resultado del análisis cerámica y la arquitectura) del material arqueológico identificado en campo, permitió definir siete sitios arqueológicos, a los que denominaremos: Sitio 1- Antonio Plaza; Sitio 2-El 
potrero; Sitio 3-Las milpas; Sitio 4-El cedral; y los sitios independientes que asignamos como sitio 42 , sitios 45 y sitio 52 .

A continuación haremos mención de las características que nos permiten categorizar, definir y delimitar a los siete sitios arqueológicos (Figura 5):

\section{Sitio 1- Antonio Plaza}

Se trata de una concentración de sitios con una distancia entre ellos menor a los $150 \mathrm{~m}$. Se define con las concentraciones ubicadas dentro de la actual comunidad de Antonio Plaza [sitio 1, sitio 2, sitio 3, sitio 4, sitio 5, sitio 6, sitio 7, sitio 8, sitio 9, sitio 10 , sitio 11 , sitio 12 , sitio 16 , sitio 17 , sitio 18 , sitio 20 , sitio 23 , sitio 33 , sitio 34, sitio 40 (La escuela), sitio 40b y 43]. Es decir, de acuerdo al estudio de patrón de asentamiento, dichas concentraciones constituyen un sólo sitio.

\section{El sitio 1- Antonio Plaza}

Se localiza a una altura menor a los $17 \mathrm{msnm}$. En consecuencia, se encuentra cercano a los terrenos inundables y a una distancia inmediata del río Uxpanapa, así como a los arroyos que en el desembocan, tal es el caso del arroyo Mariano que lleva un transcurso que atreviesa la comunidad de Antonio Plaza. Unicamente, la concentración identificada como sitio 40 durante el trabajo de campo, se caracteriza por un mónticulo modificado. Dicha estructura coincide con el supuesto lugar de hallazgo de la escultura de El Luchador, la escuela primaria. Respecto a la cerámica recolectada en los sitios que constituyen a esta 
concentración, durante el recorrido de superficie, pudimos observar que una alta densidad de material cerámico se encuentra presente en este grupo y donde descatan tipos diagnosticos relativos a los periodos Preclásico Inferior y Preclásico Medio (Symonds et al., 2002; Rust 2008; Von Nagy et al., 2002 y Coe y Diehl 1980. Destacan en número los tipos denominados como Caamaño, Capulín burdo, Garza alisado, Negro pulido con cocción diferencial (Tigrillo A y C), Tiestos con chapopote, entre otros.

\section{Sitio 2-El potrero}

Dentro de este grupo, se hallán el sitio 13 , sitio 13b, sitio 14, sitio 19, Sitio 21, sitio 22 , sitio 24 , sitio 25 , sitio 26 , Sitio 27 , sitio 31 y sitio 32 . Aunque tiene la misma distancia entre un sitio y otro que en el grupo anterior $(150 \mathrm{~m})$, se definen por ubicarse en una altura entre los $20 \mathrm{msnm}$ y los $30 \mathrm{msnm}$. Asimismo, la cerámica en superficie se caracteriza por incluir tipos de pastas finas de color naranja, crema y gris, típicas del periodo Clásico Tardío-Terminal, según la tipología establecida en otros sitios de la región (Coe y Diehl, 1980; Symonds et al. 2002). Su nombre se deriva por la única área utilizada como protero dentro del región. Asimismo, se define por arquitectura monumental, nivelaciones artificiales con forma de terrazas. 
Sitio 3-Las milpas

El sitio 3- Las milpas se constituye por el sitio 28, sitio 29, sitio 30, sitio 35, sitio 36 , sitio 37 , sitio 38 , sitio 39 , sitio 44 , sitio 46 , sitio 53 . Se caracteriza por una concentración de sitios con una distancia promedio mayor a los $200 \mathrm{~m}$ y una elevación mayor a los $30 \mathrm{msnm}$, aunque escapa el sitio 36 con una altura de 40 msnm. Podemos destacar la presencia de arquitectura monumental con plataformas artíficiales y modificación por terraceado. Dichas estructuras confluyen a un área plana dentro de la isla en la que actualmente los pobladores habilitan canales de riego que desembocan en el arroyo El Arenal. Podemos pensar que esta misma práctica pudo utilizarse en época prehispánica. Estos sitios, además de presentar una densidad de material de medio a alto, está representado por tipos diagnosticos de los periodos Preclásico Inferior, Preclásico Medio y Clásico Tardío-Terminal, de acuerdo a lo descrito en las tipologías planteadas en otros proyecto de la región.

\section{Sitio 4-El Cedral}

Este grupo ubicado al este del área de estudio, dentro del Ejido El Cedral, está constituido por nueve sitios $(41,47,48,49,50,51,54,55$ y 56), mismos que tienen una distancia promedio mayor a los $150 \mathrm{~m}$. y menor a los $350 \mathrm{~m}$; se localizan a una altura mayor a los $30 \mathrm{msnm}$, alcanzando los $40 \mathrm{msnm}$. Presenta arquitectura monumental con una forma particular, a la que le hemos llamado Tipo C o Herradura, por tratarse de estructuras semicirculares de gran tamaño. La 
cerámica recolectada en el denominado Sitio 4-El Cedral tiene una densidad con grado medio a bajo y está representada por tipos cerámicos de pastas finas, en donde destaca el anaranjado fino (Tipo Campamento), y correspondiente al Periodo Clásico tardío-terminal.

Sitios independientes: sitio 42, sitio 45 y sitio 52

Esta categorización está determinada por el sitio 42, sitio 45 y sitio 52. Se trata de concentraciones de material arqueológico aislados o una distancia mayor a los 250 de las concentracione anteriormente definidos. Los tres sitios independientes se localizan a una altura de hasta $45 \mathrm{msnm}$. La cerámica recolectada en el denominado Sitio 4-El Cedral tiene una densidad con grado medio a bajo y está representada por tipos cerámicos de pastas finas, en donde destaca el anaranjado fino (Tipo Campamento), y correspondiente al Periodo Clásico tardío-terminal.

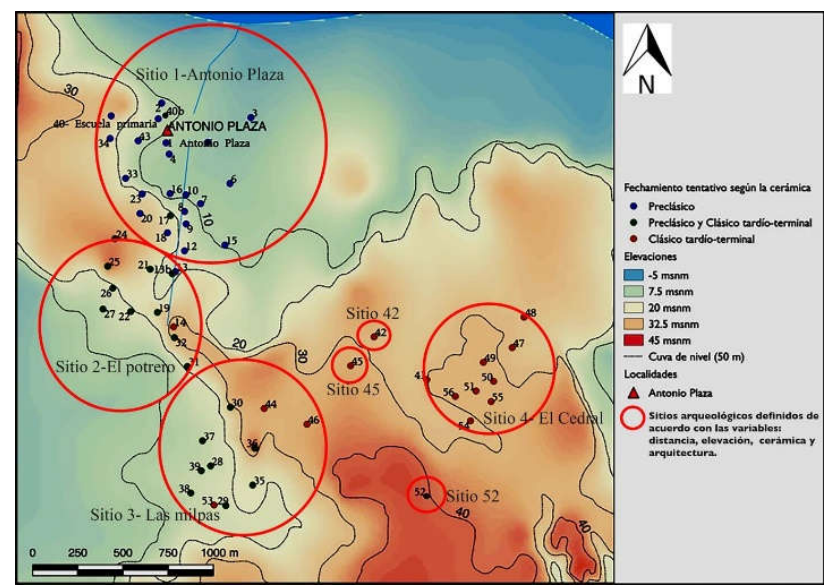

Figura 5. Imagen que muestra los siete sitios arqueológicos de la región de Capoacan, Veracruz definidos de acuerdo al análisis de los resultados de la temporada 2018. Elaborado por Virginia Arieta. 


\section{CONSIDERACIONES FINALES: LA REGIÓN DE CAPOACAN EN EL CONTEXTO DE LA REGIÓN OLMECA}

A nuestra manera de ver, el presente proyecto de investigación, aún encontrándose en su fase más temprana, muestra resultados significativos. Subrayamos de manera firme su carácter de tentativos en tanto actualmente nos encontramos en la etapa de análisis de materiales obtenidos durante la temporada de campo 2019. Asimismo, los datos tendrán que ser verificados a través de un programa de excavaciones en un futuro cercano.

Dicho lo anterior, podemos señalar en referencia a las hipótesis en las que se basa el proyecto de investigación, que: 1) La región de Capoacan, particularmente el sitio de Antonio Plaza, fue ocupado en época prehispánica, durante los periodos Preclásico y Clásico Tardío-Terminal; 2) Los resultados del análisis de materiales, particularmente en comparación con la cerámica diagnóstica de los periodos Preclásico Inferior y Medio, señalarían a Antonio Plaza como sitio olmeca con ocupación predominante durante el Preclásico Medio; 3) De acuerdo a lo anterior, podemos inferir que la escultura conocida como El Luchador, de corroborar el lugar de su hallazgo en la escuela, probablemente fue manufacturada por la sociedad olmeca de Antonio Plaza y, 4) El entorno medio ambiental juega un papel de suma importancia, una isla rodeada de terrenos inundables y un patrón de asentamiento relacionado a ríos, condicionaron en estilo de vida de los pobladores antiguos en la región. 
Los ríos representan un elemento muy importante en época prehispánica - y en la actualidad-en la costa del Golfo. Especificamente para el área olmeca, se ha planteado la posibilidad de que las corrientes fluviales sirvieran como transporte, vías de comunicación, intercambio, comercio y puertos fluviales desde el periodo Preclásico. Como señalamos desde un inicio, la isla de Capoacan tiene una ubicación estratégica respecto a las capitales olmecas de San Lorenzo y La Venta en donde los ríos Coachapa y Uxpanapa, muy posiblemente tuvieron un papel predominante en cuanto a la relación de sitios.

Consideramos que la continuación de un estudio sistemático de la región de Capoapan es de vital importancia para una mayor y mejor comprensión de la historia prehispánica de la región. De acuerdo a lo observado en la primera temporada de campo (2018) y las interpretaciones derivadas del análisis del 100\% de los materiales arqueológicos, hoy sabemos que la región estuvo poblada en época prehispánica y que Antonio Plaza fue un sitio olmeca con una ocupación durante los periodos Preclásico Inferior y Medio, lo que converge y se fortalece con su ubicación geográfica justo en medio de las dos capítales olmecas-35 km al noreste de San Lorenzo y $44 \mathrm{~km}$ al suroeste de La Venta, Tabasco.

La información obtenida hasta el momento, enfocada en la dinámica poblacional y las interacciones socio-políticas en la región de Capoacan a través de un estudio de patrón de asentamiento, no sólo ayudará a responder cuestionamientos como el de las esculturas supuestamente ahí localizadas. También, y de manera más 
importante, aportará datos que llenen vacíos sobre el conocimiento de la historia ocupacional prehispánica en la costa sur del Golfo.

\section{REFERENCIAS BIBLIOGRÁFICAS}

ALONSO, ALEJANDRA. Estudio Arqueológico en el Cerro de La Encantada, Veracruz. Tesis de Maestría en Antropología, Facultad de Filosofía y Letras, UNAM, México. 2003.

ARIETA, BAIZABAL VIRGINIA. Dinámica poblacional e interacciones sociopolíticas en la región de Capoacan, Veracruz- Reconocimiento Arqueológico Antonio Plaza. Informe Técnico Final, Archivo Técnico INAH, México. 2018.

BORSTEIN, JOSHUA A. Tripping over colosal heads: Settlement Patterns and population development in the upland Olmec heartland. Tesis doctoral, The Pennsylvannia State University, State College. 2001.

BUDAR, JIMÉNEZ, LOURDES. Informe técnico del Proyecto Piedra Labrada-Sierra de Santa Marta, Los Tuxtlas, Ver. Temporada 2014. Informe Técnico del INAH, México. 2014.

COE, MICHAEL Y MARY E. MILLER. The Olmec Wrestler: A Masterpiece of the Ancient Gulf Coast, Minerva. The International Review of Ancient Art and Archaeology, vol. 16, núm. 1. 2005.

CYPHERS, ANN. Faking Ancient Mesoamerica de Nancy L. Kelker y Karen O. Bruhns, Reseña en Anales del Instituto de Investigaciones Estéticas, Volumen XXXII, Núm. 97, p. 1-5. 2010.

CYPHERS, ANN Y ARTEMIO LÓPEZ CISNEROS. El Luchador; historia antigua y reciente, Arqueología Mexicana, vol. XV, Núm 88. p. 66-70. 2007.

CYPHERS, ANN Y ARTEMIO LÓPEZ CISNEROS. La historia de El Luchador. En María Teresa Uriarte y Rebecca González Lauck (eds.), Olmeca: balance y perspectiva. Memoria de la Primera Mesa Redonda, México, UNAM/ Consejo Nacional para la Cultura y las Artes/ Fundación Arqueológica del Nuevo Mundo, pp. 411-423. 2008. 
CYPHERS, ANN, JUDITH ZURITA Y MARCI LANE. Retos y Riesgos en la Vida Olmeca. Instituto de Investigaciones Antropológicas, UNAM. México. 2013.

CYPHERS, ANN Y KENNETH HIRTH. Transporte y producción artesanal en los albores del mundo olmeca. Instituto de Investigaciones Antropológicas, UNAM, México. 2016.

DANEELS, ANNICK. El patrón de asentamiento del periodo Clásico en la Cuenca Baja del río Cotlaxtla. Centro de Veracruz. Un estudio de caso de sociedades complejas en tierras bajas tropicales. Tesis de doctorado en Antropología. Facultad de Filosofía y Letras, UNAM, México. 2002.

DRUCKER, PHILIP Y E. CONTRERAS. Site Patterns in the Eastern Part of the Olmec Territory, Journal of the Washington Academy of Sciences, vol. 43, núm. 12. 1953.

ESQUIVAS, CHANTAL. On the Edge of Empire? Settlement changes in Chacalapan, Southern Veracruz, México, during the Classic and Postclassic Periods. Tesis de doctorado. Arts and Sciences, Boston University, Boston, MA. 2002.

GÓMEZ RUEDA, HERNANDO. Las Limas, Veracruz, y otros asentamiento prehispánicos de la región olmeca. Colección Científica, número 324, Serie Arqueológica, INAH. México. 1996.

HASSAN, FEKRI A. Demographic Archaeology. Department of Anthropology, Washington State University. Pullman, Washington. Academic Press. 1978.

HERNÁNDEZ JIMÉNEZ, LOURDES. Supervisión arqueológica Almagres 2D. Bloque I y II. Informe Técnico Final, Archivo Técnico INAH, México. 2007.

HERNÁNDEZ JIMÉNEZ, LOURDES. Supervisión arqueológica Almagres 2D. Ampliación Chalca. Informe Técnico Final, Archivo Técnico INAH, México. 2008. HERNÁNDEZ JIMÉNEZ, LOURDES. Inspección Emergente en las obras de Estudio de Reflexión Sísmica Tepetate NW-El Plan-Los Soldados. Almagres 2D. Archivo Técnico INAH, México. 2010.

HERNÁNDEZ JIMÉNEZ, LOURDES. Asentamiento Arqueológicos del municipio de las Choapas, Veracruz. Revista LuminaR. Estudios sociales y humanísticos, año 10, vol. X, Chiapas, México. 2012. 
JIMÉNEZ, DELGADO GERARDO. Control de recursos y jerarquías sociales en el territorio olmeca: El patrón de asentamiento del periodo Formativo en la Región JáltipanMinatitlán. En Ideología política y sociedad en el periodo formativo. Ensayos en homenaje al doctor David C. Grove. Edit. Ann Cyphers y Kenneth G. Hirth. UNAM, México. 2008.

KELKER, NANCY. Olmec Wrestler Revisited. Minerva. The International Review of Ancient Art and Archaeology, vol. 4, núm. 5 p. 30-31. 2004.

KELKER NANCY Y KAREN BRUHNS. Faking Ancient Mesoamerica. Walnut Creek, California, Left Coast Press. 2010.

KILLION, THOMAS W. Y JAVIER URCID. The Olmec Legacy: Cultural Continuity and Change in Mexico's Southern Gulf Coast Lowlands. Journal of Field Archaeology, Vol. 28, p. 3- 25. 2001.

KRUGER, ROBERT P. An Archaeological Survey in the Region of the Olmec, Veracruz, México. Tesis doctoral, Departmen of Anthropology, University of Pittsburgh, PA. 1996.

LOUGHLIN, MICHAEL L. Recorrido Arqueológico El Mesón. Final Report to Foundation for the Advancement of Mesoamerican Studies, Inc. FAMSI, Crystal River, FL. 2004.

LUNAGÓMEZ, ROBERTO. Patrón de asentamiento en el hinterland interior de San Lorenzo, Tenochtitlán, Veracruz. Tesis de Licenciatura en Antropología, Facultad de Antropología, Universidad Veracruzana, Xalapa. 1995.

LUNAGÓMEZ, ROBERTO. Los patrones arquitectónicos prehispánicos del sur de Veracruz: Época Clásica. Cuerpo Académico- Arqueología del paisaje y cosmovisión, Universidad Veracruzana, México. 2011.

LUNAGÓMEZ, ROBERTO. Desarrollo poblacional prehispánico en el istmo veracruzano: región de Medias Aguas. Tesis de doctorado, Facultad de Filosofía y Letras, UNAM, México. 2014.

POOL, CHRISTOPHER A. From Olmec to Epi-Olmec at Tres Zapotes, Veracruz, México. Olmec Art and Archaeology in Mesoamerica. Clark, John E. y Mary E. Pye (eds.), National Gallery of Art- Washington, p. 137-154, Yale University Press, New Haven. 2000. 
POOL, CHRISTOPHER A. Settlement Archaeology \& Political Economy at Tres Zapotes, Veracruz, Mexico. Monograph 50, Cotsen Institute of Archaeology, Unviersity of California, Los Angeles. 2003.

RUST, W. F. A Settlement Survey of La Venta, Tabasco, Mexico (tesis). Philadelphia: Department of Anthropology, University of Pennsylvania, Philadelphia (Ann Arbor: University Microfilms). 2008.

SANDERS WILLIAM, JEFFREY PARSONS Y ROBERT S. Santley. The Basin of Mexico: Ecological Processes in the Evolution of a Civilitation. Academic Press, New York. 1979.

SANTLEY, ROBERT, ROBERTO LUNAGÓMEZ, THOMAS BARRET, BOB ESTES, DANIEL WOLFMAN, PHILIP ARNOLD III. Reconocimiento Arqueológico de los Tuxtlas: Fase 2. Informe Técnico al Consejo de Arqueología, INAH, México. 1992.

SAUER, CARL O. Aboriginal Population of Northwestern Mexico. California: University of California Press. 1935.

STARK, BARBARA. Classic Period Mixtequilla, Veracruz, México: Diachronic Inferences from Residential Investigations. Institute for Mesoamerican Studies, Monograph 12. University at Albany, Albany, New York. 2001.

STIRLING, MATTHEW. Notes and News, American Antiquity, vol. 11, núm 2. 1945.

SYMONDS, STACEY C. Settlement Distribution and the Development of Cultural Complexity in the Lower Coatzacoalcos Drainage, Veracruz, México: An Archaeological Survey at San Lorenzo, Tenochtitlán. Tesis de doctorado, Vanderbilt University, Nashville. 1995.

SYMONDS STACEY, ANN CYPHERS Y ROBERTO LUNAGÓMEZ. Asentamiento Prehispánico en San Lorenzo Tenochtitlán. Coordinadora Ann Cyphers, Instituto de Investigaciones Antropológicas, UNAM, México. 2002.

VON NAGY, CHRISTOPHER L., MARY E. D. POHL, AND KEVIN O. POPE. Ceramic Chronology of the La Venta Olmec Polity: The View from San Andrés, Tabasco. Paper presented at the $67^{\text {th }}$ meeting of the Society for American Archaeology urder revision for publication. 2002. 
WENDT, CARL J. Early Formative Domestic Organization and Community Patterning in the San Lorenzo Tenochtitlán Region, Veracruz, México. Tesis de doctorado, The Pennsylvannia State University, State College. 2003.

WENDT, CARL Y ROBERTO LUNAGÓMEZ. Investigating the Arroyo Pesquero Olmec. Méxicon, Zeitschrift für Mesoamerika forschung- Revista sobre Estudios Mesoamericanos, Vol. XXXIII, Nr 3, p. 72-79, Alemania. 2011.

WILLEY, GORDON R., W. BULLARD, J. GLASS Y J. GLOFFORD. Prehistoric Maya Settlement Patterns in the Belize Valley. Papers of the Peabody Museum of Archaeology and Etnology, Volume 54, Harvard University, Cambridge. 1965. 